\title{
Train scheduling of Shinkansen and relationship to reliable train operation
}

\author{
S. Sone \& Y. Zhongping \\ Beijing Jiaotong University, China
}

\begin{abstract}
This paper explains why security is important, especially in Asia, as well as safety, and how we established reliable transportation in the Japanese Shinkansen, mainly in relation to train scheduling. The authors also describe several ideas actually taken by Shinkansen in order to realise reliable operation even in the case of possible disturbances. Out of many ideas, some examples of which are shown here, selective adoption according to the purpose of the railway or line is strongly recommended, together with given conditions taken into account.
\end{abstract}

Keywords: disturbance, punctuality, reliable operation, spare time, train scheduling.

\section{Introduction}

Features of east-Asian high-speed railways are very dense passenger flow together with frequent train operation with a big capacity. In order to realise reliable transportation in this circumstance, safe train operation in a narrow sense, which is guaranteed mainly by signalling system, is not enough; secure passenger flow must also be guaranteed even when some traffic disturbances take place. This is the reason why the authors present this paper, which mainly deals with security rather than safety, for the special invited session of "Traffic Control and Safety of High-speed Railways in Asia".

Just after the inauguration of Tokaido Shinkansen in 1964, we had many disruptions to train operation due to rain and snowfall, breakdown of the power feeding system, deterioration of track conditions due to excess axleload, etc. In a narrow sense of safety, the Japanese Shinkansen carried more than nine billion passengers without any casualty by train accident, which is by far the safest 
railway in the world. The total time spent on Shinkansen trains by all nine billion passengers exceeds 15 billion hours, which corresponds to the total lifetime of 25 thousand people. From these figures, it is not surprising that not a few people died in Shinkansen trains, from disease or even by murder, even if we exclude suicides. Trains and other accidents of the Shinkansen did happen many times, including the overrun of an empty train to the dangerous area of Tokaido Shinkansen's main line, the fall of the bridges of Sanyo Shinkansen by an earthquake, which took place just before the starting time of train operation of the day and derailment of a running train of the Joetsu Shinkansen at high-speed by another earthquake, etc, which means that there having been no casualties so far can be thought of as due to luck.

This paper deals with how we have established reliable train operation with the very heavily trafficked Japanese Shinkansen, common to east-Asian countries, in relation to train scheduling.

\section{Special features of the Shinkansen in relation to train scheduling}

\subsection{Frequent train operation with relatively few intermediate stations}

Passenger dedicated lines tend to have fewer intermediate stations in order to run trains faster than on conventional lines and the number of trains on the lines tends to be greater in order to provide better services; this means that in the case of disruption of train traffic, it is difficult or impossible to stop each train at a track facing platform.

\subsection{Uni-directional signalling system with few crossover routes}

Unlike many other high-speed railways in the world, the track layout and signalling system of the Japanese Shinkansen was modelled on the then modern double-track urban/suburban railways; uni-directional signalling, many passing loops and few crossover routes between down and up tracks.

\subsection{Existence of trains of different average speeds due to different number of intermediate stops}

Compared with commuter trains, whose acceleration and deceleration rate is high, station stopping time is short and maximum speed is low, the additional time of high-speed trains per each additional stop at intermediate station is much longer; typically four minutes in the Japanese Shinkansen against one minute for commuter trains.

In order to allocate fast and slow trains on a highly trafficked line, many passing loops are required in the Japanese Shinkansen. Indeed almost all intermediate stations have two platforms each facing one or two side lines off the main passing line. The exceptions to this on the Tokaido and Sanyo Shinkansen are only two, Atami and Shin-Kobe, at the latter of which all trains stop so that no difference of average speed takes place here. 


\subsection{Through operation to lines with different characteristics}

Different characteristics include the quality and quantity of traffic demand, and very many differences of reconstructed existing lines to accept through trains from Shinkansen: The so-called Yamagata Shinkansen and Akita Shinkansen were once narrow gauged local lines. Now the track gauge is widened to international standard, $1435 \mathrm{~mm}$, but the loading gauge, electric system, AC $20 \mathrm{kV}$, maximum speed of $130 \mathrm{~km} / \mathrm{h}$, and existence of level crossings with road traffic, etc, are still in the conventional line's standard.

\section{Problems of train operation during traffic disturbance}

\subsection{Minimum train headway by signalling system}

In normal conditions, train groups scheduled with a train headway longer (by $\boldsymbol{T}$ spare) than the theoretical minimum $(\boldsymbol{T} \min )$ can be realised stably and if a train is delayed (by $\boldsymbol{T}$ d), each following train can follow by the headway of $\boldsymbol{T} \min$, this means the initial delay can be absorbed up to $\boldsymbol{T} \mathrm{d} / \boldsymbol{T}$ spare-th train. However, in the case of some disturbance, such as a temporary speed restriction at a place, the following train can pass the same place by headway of $\boldsymbol{T} \min +\boldsymbol{T}$ r. If $\boldsymbol{T}$ r is bigger than $\boldsymbol{T}$ spare (this is not a rare case), it is often observed that the initial delay diverges and all the following trains must run at the headway of $\boldsymbol{T} \min +\boldsymbol{T}$, which is longer than the scheduled headway. It is not so easy to find the longest $\boldsymbol{T r}$ beforehand from the designed data of the signalling system and traction performance.

\subsection{Uni-directional signalling system}

Contrary to European practice, the Japanese double track section is equipped with uni-directional signalling only, with relatively few crossover routes between down and up tracks. This is a big source of problems in rescuing trains in case of a big traffic disturbance. The Tokaido Shinkansen prepared two high-power high-performance diesel locomotives for rescuing purposes, but they have never found the route to arrive at the required spot.

\subsection{More trains running at a time than the total number of tracks at intermediate stations}

In a wide area disturbance for an estimated long time, we want to stop all trains at a platform so that any passengers can get out of the train to the street, but the total number of platforms in the Tokaido Shinkansen is much smaller than the total number of trains running at a time in peak hours.

\subsection{Not enough tracks at the most important terminal station}

Shinkansen's Tokyo terminal has 10 tracks in all; six for the Tokaido Shinkansen (trains run through to Sanyo Shinkansen) and four for the Tohoku Shinkansen 
(trains run through to Joetsu, Nagano, the so-called Yamagata and Akita Shinkansen). The maximum number of revenue trains per hour per direction, excluding out-of-service trains, is 14 for Tokaido and also 14 for Tohoku.

\subsection{Sub-terminal station and required function of it in case of traffic disturbance}

Tohoku Shinkansen's Ueno station, which is next to Tokyo terminal, has four tracks all of which face to platform, and at first Tokyo terminal had only two tracks. At that time Ueno was used as a sub-terminal station effectively, some trains originate from and terminate to Ueno, and some other trains run empty between Tokyo and Ueno for arrangement to reuse as a passenger train.

The Tokaido Shinkansen has added Shinagawa sub-terminal with four tracks all facing platforms, and three additional sidings for use of draw out and storage tracks, but flexible usage in case of traffic disturbance can hardly be done by thoughtless design of the line profile; it is impossible to run between additional sidings and the Tokyo terminal.

\subsection{Cancellation of trains is difficult due to seat reallocation and keeping impartiality among passengers}

Most seats of all trains are pre-booked to passengers in Japanese practice with relatively few non-reserved seats. In this situation, even when the average loading factor is $50 \%$, no trains can easily be cancelled because of the difficulty in reallocation of pre-booked seats fairly. If cancellation of trains is inevitable by a big disruption, all pre-booked seats are cancelled and used on a first-come, first-served basis with compensation of refund of express surcharge.

\section{Train scheduling to keep in mind reliable train operation}

\subsection{Not to use connected fast and slow trains}

The scheduling pattern of "connected fast and slow trains" is very popular and reasonable for commuter railways in which a slow train arrives at a transfer station followed by arrival of the fast train at an adjacent track facing the same platform and after transfer of passengers the fast train departs first then the slow train follows. Typical timing of this procedure is; one minute after arrival of the slow train the fast train arrives and its dwelling time is also typically one minute or a little shorter, the slow train can depart about one minute after the fast train's departure. This means the dwelling time of the slow train is about three minutes.

If the same sequence is applied to a pair of high-speed trains, the following intervals of both arrival and departure are about three minutes each and the dwelling time of the slow train is about eight minutes, because the fast train stops for about two minutes. Instead of "connecting fast and slow trains", simple passing requires much shorter additional dwelling time for a slow train, which is typically 2.5 minutes, arrival to passing, plus one minute, passing to departure, minus 1.5 minutes, plus necessary dwelling time. 
From the passengers' point of view, a well-organised train schedule is convenient as a "connected" pattern.

\subsection{Reducing level crossing interference at the terminal station and intermediate important stations}

There are six tracks in the Tokyo terminal of the Tokaido Shinkansen. The track layout is the simplest one, as shown in Fig. 1. In this layout, out of 30 combinations of a pair of incoming and outgoing trains, only nine pairs have no level crossing interference and the remaining 21 pairs have interference with each other, as shown in Table 1, according to the timing. If we add the routes as shown in Fig. 2, the number of interference free pairs can be increased from nine to 15 , as shown in Table 2. (The track number is from bottom to top 14 through 19.)

In Tokyo, there is not enough space to realise Fig. 3, but train scheduling is made so as to avoid interference; arrival and departure time is restricted to prefixed times: 0 min 0 sec., 3 min. $20 \mathrm{sec}$., 6 min. $40 \mathrm{sec}$., $10 \mathrm{~min}$. $0 \mathrm{sec}$., etc. The duration of $3 \mathrm{~min} .20 \mathrm{sec}$. corresponds to the minimum train headway, including the necessary time margin. The Tokyo station of the Tohoku Shinkansen has similar prefixed timing of 4 minute intervals.

This is a very easy way to avoid interference and is effective if trains run exactly enough and even if trains are much delayed, this pattern can be applied, although it is not the best way.

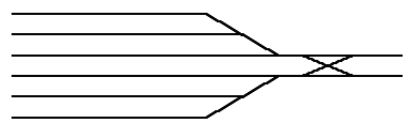

Figure 1: Existing layout.

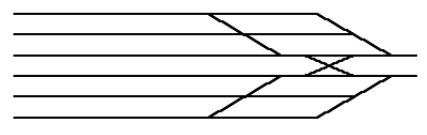

Figure 2: Improved layout.

\begin{tabular}{|c|c|c|c|c|}
\hline Tabl & e 1: & $\begin{array}{l}\text { Interference } \\
\text { of Fig. } 1 .\end{array}$ & $\begin{array}{l}\text { Interference } \\
\text { of Fig. } 2 .\end{array}$ & \\
\hline $\begin{array}{l}\text { out } \\
\text { in }\end{array}$ & 1415 & 16171819 & $\begin{array}{l}\text { out } 141516171819 \\
\text { in }\end{array}$ & \\
\hline 14 & $-x$ & $x 000$ & $14-00000$ & $\bigcirc$ is an interference \\
\hline 15 & $\mathrm{x}-$ & $x \bigcirc 00$ & $15 x-\bigcirc \bigcirc \bigcirc 0$ & free route while $\mathrm{x}$ \\
\hline 16 & $\mathrm{x} x$ & -000 & $16 x \times-000$ & route interferes with \\
\hline 17 & $\mathrm{x} x$ & $x-x x$ & $17 \times x \times-00$ & ath \\
\hline 18 & $\mathrm{x} x$ & $x \quad x-x$ & $18 \times \times \times x-0$ & \\
\hline 19 & $\mathrm{x} x$ & $\mathrm{x} x \mathrm{x}-$ & $19 \times \times \times x \times-$ & \\
\hline
\end{tabular}


Much better practice can be observed at private railway commuter lines, such as the Odakyu Electric Railway's Shinjuku terminal: 5 track terminal of two levels in which interference takes place very seldom.

\subsection{Various ideas to prevent disturbance from divergence}

At the Fukushima station of the Tohoku Shinkansen, only one track out of four is used for thorough operation to and from the so-called Yamagata Shinkansen. The seven car Yamagata Shinkansen trains leave Tokyo coupled with the eight car Tohoku Shinkansen train for Sendai and in reverse the Yamagata Shinkansen train couples with the Tohoku Shinkansen train from Sendai on the same track. Even in case of disturbance, coupling and decoupling cannot be made at the same time. The required time to couple/decouple trains is much longer than normal station dwelling time, so it is convenient to be passed by the fast train, Hayate, during this stopping time. This requirement of train scheduling is the biggest restriction of the whole Tohoku Shinkansen train scheduling.

\subsection{Train crossing at the intended partial double-track section on the Akita Shinkansen}

Between Omagari and Akita, 51.7km, of the so-called Akita Shinkansen was once a double track section of narrow gauge Ouu line. This section was converted to two single line tracks in parallel, one narrow gauge and the other standard gauge. However, out of this section, a $12.4 \mathrm{~km}$ section between Jinguuji and Mineyoshigawa is laid of one standard gauge track in parallel with a dual gauge track so that standard gauge trains can pass each other while running. This particular section was chosen from the train schedule of the Akita Shinkansen where trains cross each other, plus a margin for the possible delay of one train.

\subsection{Enough spare time allocated on the Tokaido Shinkansen in highly trafficked hours}

The fastest Nozomi trains using the newest Series N700 trainset can run between Tokyo and Shin-Osaka in 2 hours 25 minutes with reasonable spare time. Actually, three trains are scheduled to run in this time in early morning and late at night, but during most hours of the day the Nozomi trains, exclusively the N700 trainset, run in an additional 8 to 12 minutes, which is partly necessary due to mixed traffic with slower trains and partly due to giving a recovery margin in case of train delay.

Whether an additional 8 to 12 minutes on top of the basic 145 minutes is justifiable or not may be a big question.

\section{Conclusion}

The authors do not think all of these practice are necessary or inevitable because there are many other countermeasures to keep punctuality or to avoid large disturbances, as seen in private railways lines. For instance, the average required 
time of 155 minutes between Tokyo and Shin-Osaka, 10 minutes longer than the basic time, seems too long if the competitive situation with airlines is taken into account.

The Akita Shinkansen's partial double track section of standard gauge line is very effectively used at the moment, when trains run regularly at 60 minute intervals. This means it is difficult to add trains flexibly without substantially longer travelling time.

Fukushima's case of coupling (of up trains) and decoupling (of down trains) on only one track requires too much restriction to the whole train schedule of Tohoku Shinkansen. Under this track layout, trains from Sendai must cross down the main line twice to couple with the Akita Shinkansen train; this is too restrictive to train operation in the case of disturbance. Another measure to cope with this situation, such as to provide a new route from the Yamagata Shinkansen to the scarcely used track 11 of the Fukushima station, where up trains can couple, should be taken even if the new route crosses down the main line.

In east-Asian countries, frequent train operation is required mainly to realise the large capacity, while in European countries, this is required mainly to realise better connections between trains. From this difference, frequent train operation in Asia should accompany reliable train operation, especially in peak demand hours.

Necessary techniques for this may be different line by line or time by time: The authors recommend selective application by each high-speed railway section according to the purpose of the line and time, rather than to take the proven best practice from a line of different purpose.

\section{References}

[1] Timetable of Shinkansen: issued every month in Japan (in Japanese) by several publishers; bi-monthly by Thomas Cook Publishing UK as Overseas Timetable.

[2] Track layout of railways: officially undisclosed by railways; but few private enthusiasts published so far including Tokaido Lines, by Ryozo Kawashima from Kodansha Publishing Co. (in Japanese) and Quail Map Series, by John Yonge from Quail Map Co. Exeter, UK.

[3] Detailed list of rolling stock for each line or railway; edited by JRR, issued semi-annually for Japan Railways Group and annually for Japanese Private Railways Group by Kotsu-shimbunsha. (in Japanese)

[4] Tracks of each station including normal operation practice: http://ja. wikipedia.org/wiki/[station name in kanji such as 東京駅] 